\title{
Enteric Glia Are Targets of the Sympathetic Innervation of the Myenteric Plexus in the Guinea Pig Distal Colon
}

\author{
Brian D. Gulbransen, Jaideep S. Bains, and Keith A. Sharkey \\ Hotchkiss Brain Institute, Department of Physiology and Pharmacology, University of Calgary, Calgary, Alberta T2N 4N1, Canada
}

Astrocytes respond to synaptic activity in the CNS. Astrocytic responses are synapse specific and precisely regulate synaptic activity. Glia in the peripheral nervous system also respond to neuronal activity, but it is unknown whether glial responses are synapse specific. We addressed this issue by examining the activation of enteric glia by distinct neuronal subpopulations in the enteric nervous system. Enteric glia are unique peripheral glia that surround enteric neurons and respond to neuronally released ATP with increases in intracellular calcium $\left(\left[\mathrm{Ca}^{2+}\right]_{\mathrm{i}}\right.$ ). Autonomic control of colonic function is mediated by intrinsic (enteric) and extrinsic (sympathetic, parasympathetic, primary afferent) neural pathways. Here we test the hypothesis that a defined population of neurons activates enteric glia using a variety of techniques to ablate or stimulate components of the autonomic innervation of the colon. Our findings demonstrate that, in the male guinea pig colon, activation of intrinsic neurons does not stimulate glial $\left[\mathrm{Ca}^{2+}\right]_{\mathrm{i}}$ responses and fast enteric neurotransmission is not necessary to initiate glial responses. However, ablating extrinsic innervation significantly reduces glial responses to neuronal activation. Activation of primary afferent fibers does not activate glial $\left[\mathrm{Ca}^{2+}\right]_{\mathrm{i}}$ responses. Selectively ablating sympathetic fibers reduces glial activation to a similar extent as total extrinsic denervation. Neuronal activation of glia follows the same frequency dependence as sympathetic neurotransmitter release, but the only sympathetic neurotransmitter that activates glial $\left[\mathrm{Ca}^{2+}\right]_{\mathrm{i}}$ responses is ATP, suggesting that sympathetic fibers release ATP to activate enteric glia. Therefore, enteric glia discern activity in adjacent synaptic pathways and selectively respond to sympathetic activation.

\section{Introduction}

The concept of neuron-glia chemical signaling is slowly gaining acceptance as a fundamental principle of nervous system function. Glia, or more specifically, in terms of synaptic function, astrocytes, express receptors for multiple ligands and, once activated, can quickly recruit neighboring glia either via paracrine signals or through gap junctions (Kettenmann and Ransom, 2004). This apparently indiscriminate signaling capacity is at odds with our current views of nervous system function, specifically, that neural regulation is both temporally and spatially precise. One resolution to this impasse is found in recent work demonstrating that activation of discrete subdomains of individual astrocytes can signal independently to control the strength of neuronal synapses in their immediate vicinity (Gordon et al., 2009). This suggests that glia have the capacity for differential signal transduction that is consistent with unique neuronal targeting.

Outside of the CNS, the concepts of neuron-glial signaling are less well defined. Autonomic nerves release multiple chemical

Received Feb. 3, 2010; revised March 8, 2010; accepted March 24, 2010.

This work was supported by a grant from the Canadian Institutes of Health Research (CIHR) (K.A.S.). B.D.G. holds fellowships from the Canadian Association of Gastroenterology (CAG)/CIHR/Crohn's and Colitis Foundation of Canada (CCFC) (fellowship award) and Alberta Heritage Foundation for Medical Research (AHFMR). K.A.S. is an AHFMR Medical Scientist and holds the CCFC Chair in Inflammatory Bowel Disease Research at University of Calgary. J.S.B. is an AHFMRSenior Scholar. We thank Michelle Thacker for technical assistance during extrinsic denervation surgeries.

Correspondence should be addressed to Brian D. Gulbransen, Hotchkiss Brain Institute, Department of Physiology and Pharmacology, University of Calgary, 3330 Hospital Drive NW, Calgary, Alberta T2N 4N1, Canada. E-mail: bgulbran@ucalgary.ca.

DOI:10.1523/JNEUROSCI.0603-10.2010

Copyright $\odot 2010$ the authors $\quad 0270-6474 / 10 / 306801-09 \$ 15.00 / 0$ messengers (Burnstock, 2009), and it is not clear whether glia are targeted by specific transmitters or specific populations of autonomic nerves. We have addressed this issue by examining the activation of enteric glia by distinct neuronal subpopulations in the enteric nervous system (ENS). The ENS is particularly well suited for this type of work because neuron-glia interactions can be studied in situ using preparations with intact synaptic pathways from four main neuronal populations that can be selectively stimulated or eliminated (Furness, 2006). Within the ENS, neurons and glia are tightly packed into ganglia that are interconnected by nerve fiber bundles containing the axons of intrinsic (enteric) as well as extrinsic (sympathetic, parasympathetic, primary afferent) neurons. Enteric glia surround enteric synapses and share many morphological, molecular, and electrophysiological features with astrocytes (Gabella, 1981; Jessen and Mirsky, 1983; Hanani and Reichenbach, 1994). Importantly, like astrocytes, enteric glia are active participants at enteric synapses and respond to neuronal activity (Gomes et al., 2009; Gulbransen and Sharkey, 2009). Our previous data demonstrate that electrical stimulation of axons in interganglionic nerve fiber bundles releases ATP from neurons that stimulates $\left[\mathrm{Ca}^{2+}\right]_{i}$ responses in enteric glia in situ (Gulbransen and Sharkey, 2009).

Although glia are recruited by neuronal activation, the specificity of this recruitment has not been examined. To determine which neuronal element(s) of the ENS signals to enteric glia, we used a combination of techniques to selectively stimulate or eliminate intrinsic and extrinsic populations of neurons and assayed glial activation using calcium imaging. Given that astrocytes have the capacity to selectively respond to activity at specific synapses, 
we hypothesized that a defined population of neurons releases ATP that activates enteric glia. We find that enteric glia are specifically activated by sympathetic postganglionic neurons innervating the colonic myenteric plexus, supporting the view that glia are not indiscriminate detectors of neuronal activity and can discern activity in specific neural pathways.

\section{Materials and Methods}

Animals. Animal protocols were approved by the University of Calgary Animal Care Committee and were performed in accordance with the guidelines of the Canadian Council on Animal Care. Male albino guinea pigs (225-300 g; Charles River) were used for all studies. Animals were housed in plastic sawdust floor cages and had access to Purina Laboratory Chow and water ad libitum.

Tissue preparation. Tissue was prepared as described by Gulbransen and Sharkey (2009) with only minor modifications. Briefly, animals were deeply anesthetized with halothane (5\% in oxygen; Benson Medical Industries), and a small segment of distal colon was removed and placed in ice-cold oxygenated $\left(95 \% \mathrm{O}_{2}, 5 \% \mathrm{CO}_{2}\right)$ physiological saline solution (PSS). Tissue was transferred to a Sylgard (Dow-Corning)-coated Petri dish, opened along the mesenteric border, and pinned flat, and the mucosa, submucosa, and circular muscle were removed with forceps to expose the myenteric plexus. The resulting longitudinal muscle myenteric plexus (LMMP) preparation was transferred to a Sylgard-coated, open diamond bath recording chamber, pinned flat, and incubated for 15 min at room temperature in an enzyme mixture consisting of 156.25 $\mathrm{U} / \mathrm{ml}$ Collagenase type II (Invitrogen Canada) and $1 \mathrm{U} / \mathrm{ml}$ Dispase II (Roche) dissolved in PSS with $95 \% \mathrm{O}_{2}, 5 \% \mathrm{CO}_{2}$ gently blowing across the surface of the buffer. Gentle tituration with PSS removed excess muscle and connective tissue and stopped enzyme activity. LMMPs were loaded in the dark for $45 \mathrm{~min}$ at $37^{\circ} \mathrm{C}$ (with oxygenation) with $4 \mu \mathrm{mol} / \mathrm{L}$ Fluo- 4 AM (Invitrogen), 0.02\% Pluronic F-127 (Invitrogen), and $200 \mu \mathrm{mol} / \mathrm{l}$ water-soluble Probenecid (Invitrogen) in PSS. LMMPs were then rinsed in PSS and sat for $20 \mathrm{~min}$ to de-esterify before imaging.

$\mathrm{Ca}^{2+}$ imaging. Images were acquired every $1-2 \mathrm{~s}$ through the $20 \times$ water-immersion objective [UMPlanFl, 0.5 numerical aperture (NA)] of an upright Olympus BX61WI motorized fixed-stage microscope using Imaging Workbench 6 software (INDEC BioSystems) and a CCD Hamamatsu ORCA-ER digital camera (Hamamatsu Photonics). Excitation/emission wavelengths were 485/525 nm for Fluo-4. LMMPs were continuously perfused with PSS prewarmed to near $33^{\circ} \mathrm{C}$ using a gravity flow perfusion system (Automate Scientific) and custom-made laminar flow open diamond bath perfusion chambers at $\sim 2-3 \mathrm{ml} / \mathrm{min}$.

Fiber tract stimulation. Synaptic activation of neurons was elicited by placing a concentric bipolar microelectrode, insulated except at the tip (World Precision Instruments), on an interganglionic fiber tract one ganglia away from the ganglion being imaged and delivering a train of 0.5 ms, $5 \mathrm{~V}, 2,10$, or $20 \mathrm{~Hz}$ pulse of current for $3 \mathrm{~s}$ with a Grass SD9 stimulator (Grass Technologies, Astro-Med).

6-Hydroxydopamine chemical sympathectomy. 6-Hydroxydopamine (6-OHDA) treatment was performed as described by Gershon et al. (1990). Guinea pigs received two subcutaneous injections ( $24 \mathrm{~h}$ apart) of $100 \mathrm{mg} / \mathrm{kg}$ 6-OHDA (Sigma-Aldrich) dissolved in $\mathrm{H}_{2} \mathrm{O}$ with $0.1 \%$ sodium metabisulfite as a stabilizing agent. Tissue was taken $\sim 17 \mathrm{~h}$ after the last injection. Loss of tyrosine hydroxylase (TH)-immunoreactive (IR) nerve fibers in the myenteric plexus was taken as verification of effectiveness of 6-OHDA treatment.

Surgical denervation of the colon. Extrinsic denervation was accomplished using the surgical technique described by Messenger and Furness (1992) and Lomax et al. (2000). Before surgery, guinea pigs were sedated with Diazepam ( $5 \mathrm{mg} / \mathrm{kg}$, i.p.; Sandoz Canada) and injected with the analgesic Metacam (meloxicam) (1 mg/kg, s.c.; Boehringer Ingelheim) and the antibacterial Baytril (enrofloxacine) $(5 \mathrm{mg} / \mathrm{kg}$, s.c.; Bayer, Animal Health Division). Animals were then anesthetized with an intramuscular injection of $50 \mathrm{mg} / \mathrm{kg}$ ketamine and $5 \mathrm{mg} / \mathrm{kg}$ xylazine, shaved, and cleaned with $70 \%$ ethanol $(\mathrm{EtOH})$ and betadine, and a loop of distal colon was exposed through an abdominal incision. The colonic nerves associated with the mesenteric blood vessels supplying the section of distal colon were severed, the surrounding mesentery was cut, and the artery was lightly swabbed with $80 \%$ phenol. The site of denervation was marked by a piece of surgical thread tied around the main arteriole, the colon was returned to the abdominal cavity, and the incision was sutured closed. Animals were maintained on a heating pad until the following day when they were returned to their home cage and were used 7-8 d after surgery to allow degeneration of the severed axons. As with 6-OHDA treatment, loss of TH-IR nerve fibers in the myenteric plexus was taken as verification of effectiveness of surgical denervation.

Whole-mount immunohistochemistry. Immunohistochemistry was performed as described by Nasser et al. (2007). Briefly, LMMP whole mounts were prepared from Zamboni's fixed tissue, and primary antibodies to TH (rabbit anti-TH, 1:1000; Eugene Biotech) and protein gene product 9.5 (PGP) (mouse anti-PGP, 1:500; Ultraclone) were applied for $48 \mathrm{~h}$ at $4^{\circ} \mathrm{C}$. TH was detected with the secondary antibody donkey antirabbit cyanine 3 (1:300; Jackson ImmunoResearch, BioCAN Scientific) and PGP9.5 with goat anti-mouse Alexa 488 (1:200; Invitrogen).

Confocal microscopy. Immunofluorescence was imaged on an Olympus FluoView FV1000 confocal microscope (Olympus America) using either a $40 \times($ UPlan FLN, $1.30 \mathrm{NA}$ ) or $60 \times$ (PlanApo N, $1.42 \mathrm{NA}$ ) oil-immersion lens. Optical sections $(1 \mu \mathrm{m})$ were acquired through each field of view and then compiled into a $z$-stack.

Solutions. PSS contained $135 \mathrm{mmol} / \mathrm{L} \mathrm{NaCl}, 5 \mathrm{mmol} / \mathrm{L} \mathrm{KCl}, 2.5 \mathrm{mmol} / \mathrm{L}$ $\mathrm{CaCl}_{2}, 1.2 \mathrm{mmol} / \mathrm{L} \mathrm{MgCl}_{2}, 10 \mathrm{mmol} / \mathrm{L}$ glucose, $10 \mathrm{mmol} / \mathrm{L}$ HEPES, 5 $\mathrm{mmol} / \mathrm{L}$ sodium bicarbonate, and $1 \mathrm{mmol} / \mathrm{L}$ pyruvic acid (all from SigmaAldrich), $\mathrm{pH}$ adjusted to 7.4 with $\mathrm{NaOH}$, with $3 \mu \mathrm{mol} / \mathrm{L}$ nicardipine (SigmaAldrich) and $1 \mu \mathrm{mol} / \mathrm{L}$ scopolamine (Sigma-Aldrich) to inhibit smooth muscle contractions. ATP, epibatidine, norepinephrine (NE), neuropeptide Y (NPY), somatostatin (SST), and hexamethonium (Sigma-Aldrich) were dissolved in buffer and bath applied. Capsaicin (Sigma-Aldrich) stock was prepared in $100 \% \mathrm{EtOH}$ and then dissolved in buffer (final concentration of $\mathrm{EtOH}$ was $0.01 \%$ ).

Data analysis. Enteric glia were identified as described by Gulbransen and Sharkey (2009). Only glia within myenteric ganglia were analyzed in this study. Regions of interest (ROIs) were drawn and relative fluorescence intensity was measured in Imaging Workbench 6 (INDEC BioSystems). The change in fluorescence after background subtraction was calculated using a modification of the method described by Takahashi et al. (1999) with $\Delta F / F=\left(\left(F_{1}-F_{0}\right) / F_{0}\right)_{\mathrm{ROI}}-\left(\left(F_{1}-F_{0}\right) / F_{0}\right)_{\text {background }}$, where $F_{1}$ is the fluorescence at any given point and $F_{0}$ is the baseline fluorescence. This modification was necessary to reduce noise from fluorescence changes in the muscle layer underlying the myenteric plexus. Analysis and generation of traces were performed using Prism 4 software (Graphpad Software). Traces represent the mean \pm SEM response of all glial or neuronal ROIs within a single ganglion. Statistical differences were determined by either ANOVA or $t$ test analysis with a $p$ value of $<0.05$ considered significant.

Responses were determined as increases in intracellular calcium $>3$ SDs from baseline (calculated as the average ratio during the $30 \mathrm{~s}$ preceding stimulus application). Responses are represented as the percentage of the $100 \mu \mathrm{mol} / \mathrm{L}$ ATP response in the same ganglion except the frequency range data that are expressed as the percentage of the response at $20 \mathrm{~Hz}$. Values are reported as mean \pm SEM.

\section{Results}

Enteric ganglia are innervated by enteric, sympathetic, parasympathetic, and primary afferent neurons whose axons travel between enteric ganglia in interganglionic fiber tracts. Electrical stimulation of all nerves within an interganglionic fiber tract elicits $\left[\mathrm{Ca}^{2+}\right]_{\mathrm{i}}$ responses in enteric glia mediated by neuronal ATP (Gulbransen and Sharkey, 2009). All neuronal types innervating the enteric nervous system have the potential to release ATP so it is unknown whether glia respond to general neuronal activity or whether activity in a specific pathway specifically targets glia. To determine whether a specific subset of neurons signals to enteric glia, we used pharmacological and surgical techniques to selectively activate or eliminate each population of neurons innervat- 

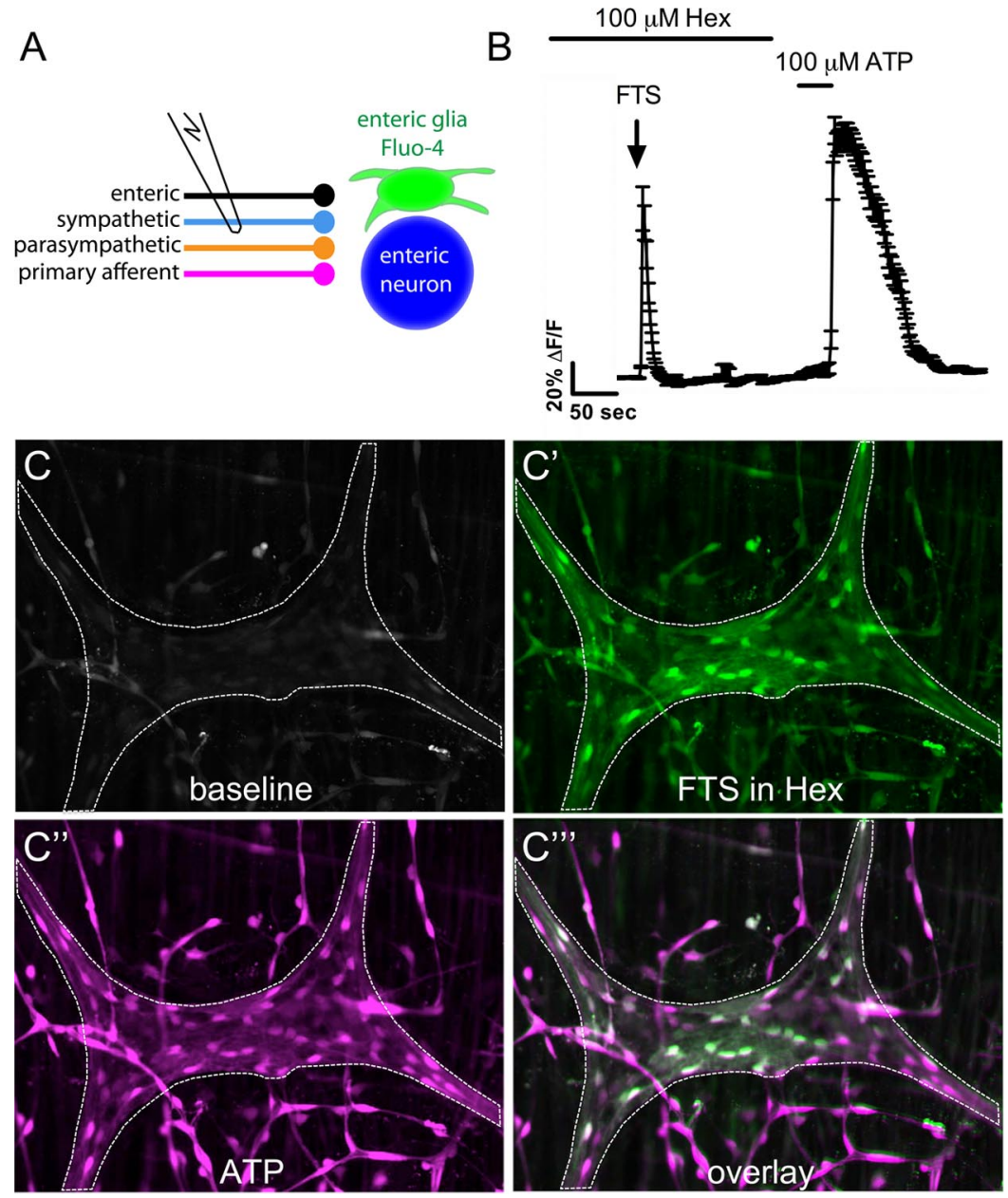

Figure 1. Electrical stimulation of interganglionic myenteric fiber tracts does not require fast nicotinic transmission to stimulate $\left[\mathrm{Ca}^{2+}\right]_{\mathrm{i}}$ responses in enteric glia. $\boldsymbol{A}$, Schematic of experiment. FTS activates enteric, sympathetic, parasympathetic, and primary afferent nerves, which then release substances that stimulate responses in enteric glia and enteric neurons. $\boldsymbol{B}$, Averaged glial response (mean \pm SEM) of 44 glial regions within a myenteric ganglion illustrates that glial responses to FTS are not blocked by hexamethonium (Hex). C, At rest (baseline), a myenteric ganglion in an LMMP whole-mount preparation from the distal colon of a guinea pig (outlined by dashed line) displays low Fluo-4 fluorescence. $C^{\prime}$, After a 5 min blockade of nicotinic receptors with 100 $\mu \mathrm{m}$ hexamethonium, electrical stimulation $(3 \mathrm{~s}, 5 \mathrm{~V}, 20 \mathrm{~Hz})$ of an interganglionic fiber tract stimulates $\mathrm{Ca}^{2+}$ responses in a proportion of glia within the ganglion (peak response pseudocolored in green). $\boldsymbol{C}^{\prime \prime}$, Bath application of $100 \mu \mathrm{m}$ ATP elicits a robust glial $\mathrm{Ca}^{2+}$ response (peak response pseudocolored in magenta). $\boldsymbol{C}^{\prime \prime}, 0$ verlay of the peak FTS and peak ATP responses demonstrates that many glia respond to both ATP and FTS.

ing the myenteric plexus and examined the effect on glial $\left[\mathrm{Ca}^{2+}\right]_{\mathrm{i}}$ responses.

\section{Cholinergic activation of enteric neurons}

The activation of nicotinic receptors is necessary for fast intrinsic synaptic communication in the myenteric plexus (Galligan and Bertrand, 1994). To determine whether ACh released from the nerve endings of interganglionic fibers is necessary to elicit changes in glial $\left[\mathrm{Ca}^{2+}\right]_{\mathrm{i}}$, we examined the effects of the nicotinic receptor antagonist hexamethonium on glial $\left[\mathrm{Ca}^{2+}\right]_{i}$ responses to electrical stimulation of the interganglionic fiber tracts (FTS). Hexamethonium $(100 \mu \mathrm{M})$ had no effect on FTS induced $\left[\mathrm{Ca}^{2+}\right]_{\mathrm{i}}$ responses in enteric glia (Fig. $1 \mathrm{~B}, \mathrm{C}$ ). Average glial responses to FTS under control conditions (42.0 \pm $10.1 \%, n=26$ ganglia) are not significantly different from glial FTS responses in the presence of hexamethonium (37.2 \pm $6.5 \%, n=19$ ganglia) (two-tailed $t$ test, $p=0.71$ ). This observation indicates that nicotinic transmission is not required for FTS activation of enteric glia.
Although nicotinic transmission between enteric neurons is not necessary for glial activation, direct cholinergic activation of enteric neurons could release substances that activate enteric glia (White, 1982). To test whether activation of enteric neurons leads to glial $\left[\mathrm{Ca}^{2+}\right]_{\mathrm{i}}$ responses, we stimulated enteric neurons with the nicotinic agonist epibatidine (300 nM). Bath application of epibatidine at slow perfusion speeds slowly raises the concentration of drug, desensitizing nicotinic receptors before responses are initiated (data not shown). To prevent desensitization of nicotinic receptors, the flow rate of the perfusion system was increased to speed delivery and removal of epibatidine. Fast application of epibatidine initiates robust and repeatable $\left[\mathrm{Ca}^{2+}\right]_{\mathrm{i}}$ responses in enteric neurons (Fig. $2 A, B)$. Epibatidine is a more effective stimulant of enteric neurons than ATP, stimulating peak $\left[\mathrm{Ca}^{2+}\right]_{\mathrm{i}}$ responses that are on average $63.2 \pm 20 \%$ larger than peak neuronal $\left[\mathrm{Ca}^{2+}\right]_{\mathrm{i}}$ responses to ATP ( $n=22$ ganglia, 4 animals). We confirmed the specificity of epibatidine for nicotinic receptors by blocking neuronal epibatidine responses with the nicotinic receptor antagonist hexamethonium (100 $\mu \mathrm{M}$ ) (Fig. 2B).

Although epibatidine stimulates large $\left[\mathrm{Ca}^{2+}\right]_{\mathrm{i}}$ responses in enteric neurons, only very small $\left[\mathrm{Ca}^{2+}\right]_{\mathrm{i}}$ responses are detected in enteric glia. Of the 22 myenteric ganglia challenged with epibatidine, glia in 19 responded significantly above baseline. On average, epibatidine stimulates peak $\left[\mathrm{Ca}^{2+}\right]_{\mathrm{i}}$ responses in glia that are $7.2 \pm 0.9 \%$ as large as ATP responses $(n=$ 22 ganglia, 4 animals) (Fig. $2 A, B$ ). This small response may be attributable to responses in neuronal elements running over or under glial ROIs in the preparation because no obvious glia $\left[\mathrm{Ca}^{2+}\right]_{\mathrm{i}}$ responses were observed during these experiments. Therefore, cholinergic nicotinic activation of enteric neurons is not sufficient to elicit glial $\left[\mathrm{Ca}^{2+}\right]_{i}$ responses of a similar magnitude to those generated by FTS.

\section{Stimulation of primary afferent nerves}

Activation or blockade of intrinsic enteric pathways had no effect on glial $\left[\mathrm{Ca}^{2+}\right]_{\mathrm{i}}$ responses. Therefore, we hypothesized that extrinsic neurons signal to enteric glia. First, we asked whether stimulation of primary afferent nerves could activate glial $\left[\mathrm{Ca}^{2+}\right]_{\mathrm{i}}$ responses. In the enteric nervous system, primary afferent nerves can be differentiated from other neuronal types by the expression of TRPV1 receptors. The majority of colonic afferents are TRPV1 immunoreactive, and stimulation of TRPV1 receptors initiates action potentials and/or calcium influx in sensory neurons (Robinson et al., 2004; Brierley et al., 2005; Malin et al., 2009). Restricted expression of TRPV1 allowed us to selectively stimulate primary afferent nerves with the TRPV1 agonist capsaicin $(50 \mu \mathrm{M})$. 

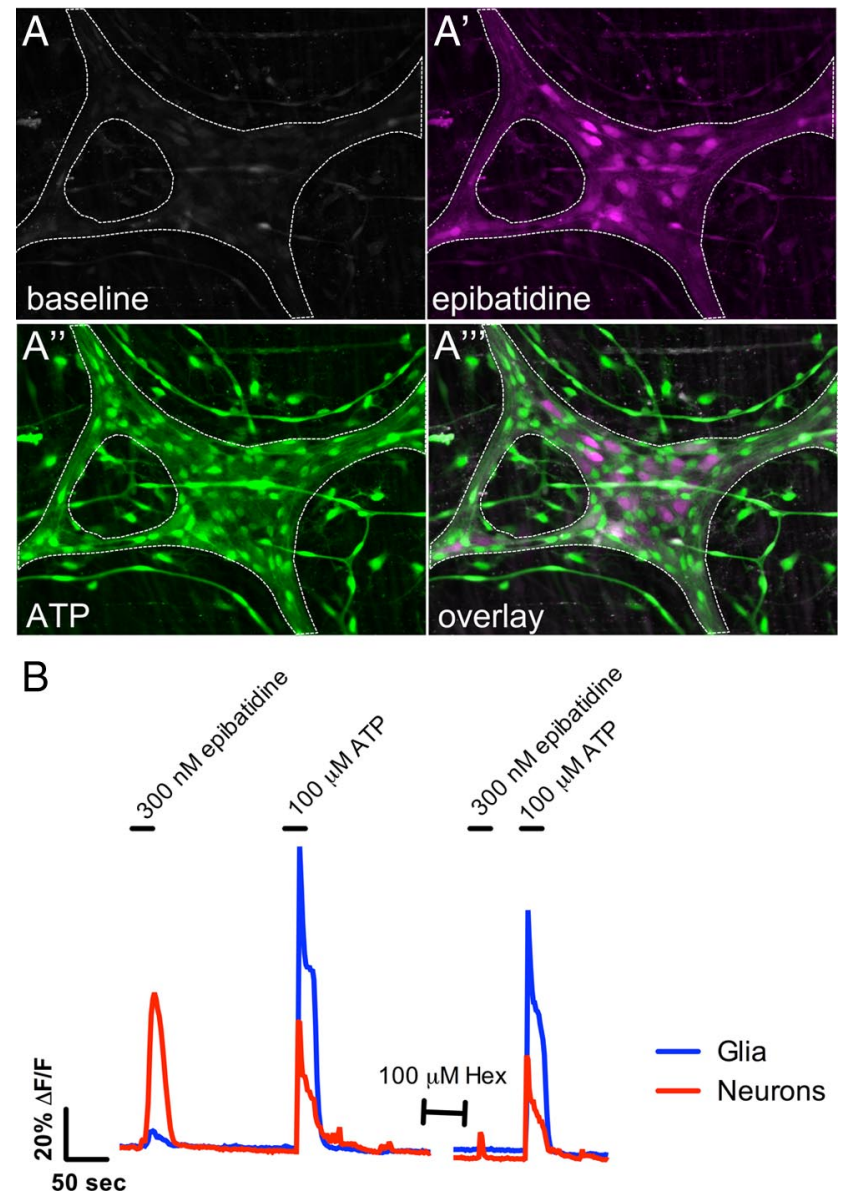

Figure 2. Nicotinic stimulation of enteric neurons is not sufficient to elicit $\mathrm{Ca}^{2+}$ responses in enteric glia. $\boldsymbol{A}$, A myenteric ganglion (outlined by dashed line) displays low Fluo-4 fluorescence at rest. $A^{\prime}$, Bath application of the nicotinic agonist epibatidine ( $300 \mathrm{~nm}$ ) elicits a robust $\mathrm{Ca}^{2+}$ response in enteric neurons (peak response pseudocolored magenta), whereas ATP elicits a $\mathrm{Ca}^{2+}$ response dominated by enteric glia (peak response pseudocolored green) $\left(A^{\prime \prime}\right) . A^{\prime \prime \prime}, 0$ verlay of the epibatidine response $\left(\boldsymbol{A}^{\prime}\right)$ and ATP response $\left(\boldsymbol{A}^{\prime \prime}\right)$ demonstrates that ATP-responding glia do not respond to nicotinic stimulation of neurons with $\mathrm{Ca}^{2+}$ responses. $\boldsymbol{B}$, Trace showing the averaged response of enteric glia ( 35 cells; blue) and neurons ( 6 cells; red) within a myenteric ganglion (error bars omitted for clarity). Neurons respond to nicotinic stimulation with robust $\mathrm{Ca}^{2+}$ responses that are inhibited by $100 \mu \mathrm{m}$ hexamethonium (Hex). Only very small $\mathrm{Ca}^{2+}$ are detected in enteric glia, possibly as a result of overlapping neuronal elements.

Bath application of capsaicin stimulates fast, transient $\left[\mathrm{Ca}^{2+}\right]_{\mathrm{i}}$ responses in enteric neurons (Fig. 3). Neuronal $\left[\mathrm{Ca}^{2+}\right]_{\mathrm{i}}$ responses to capsaicin are smaller in amplitude than neuronal $\left[\mathrm{Ca}^{2+}\right]_{\mathrm{i}}$ responses simulated by ATP, and, on average, capsaicin stimulates peak $\left[\mathrm{Ca}^{2+}\right]_{\mathrm{i}}$ responses $30.5 \pm 8.8 \%$ as large those initiated by ATP ( $n=10$ ganglia, 4 animals) (Fig. 3). Capsaicin also initiates muscle contractions in the layer of longitudinal muscle behind the myenteric plexus in our preparations. However, capsaicin does not stimulate glial $\left[\mathrm{Ca}^{2+}\right]_{\mathrm{i}}$ responses significantly above vehicle control ( $n=19$ ganglia, 4 animals; ANOVA, $p>0.05$ ) (Fig. 3).

\section{Chemical sympathectomy}

Because activation of enteric neurons and extrinsic primary afferent nerves did not activate enteric glial $\left[\mathrm{Ca}^{2+}\right]_{\mathrm{i}}$ responses, we hypothesized that ATP is released from extrinsic autonomic neurons. In the guinea pig distal colon, the majority of extrinsic innervation is from sympathetic adrenergic nerves (Olsson et al., 2006). To determine the contribution of sympathetic nerves in
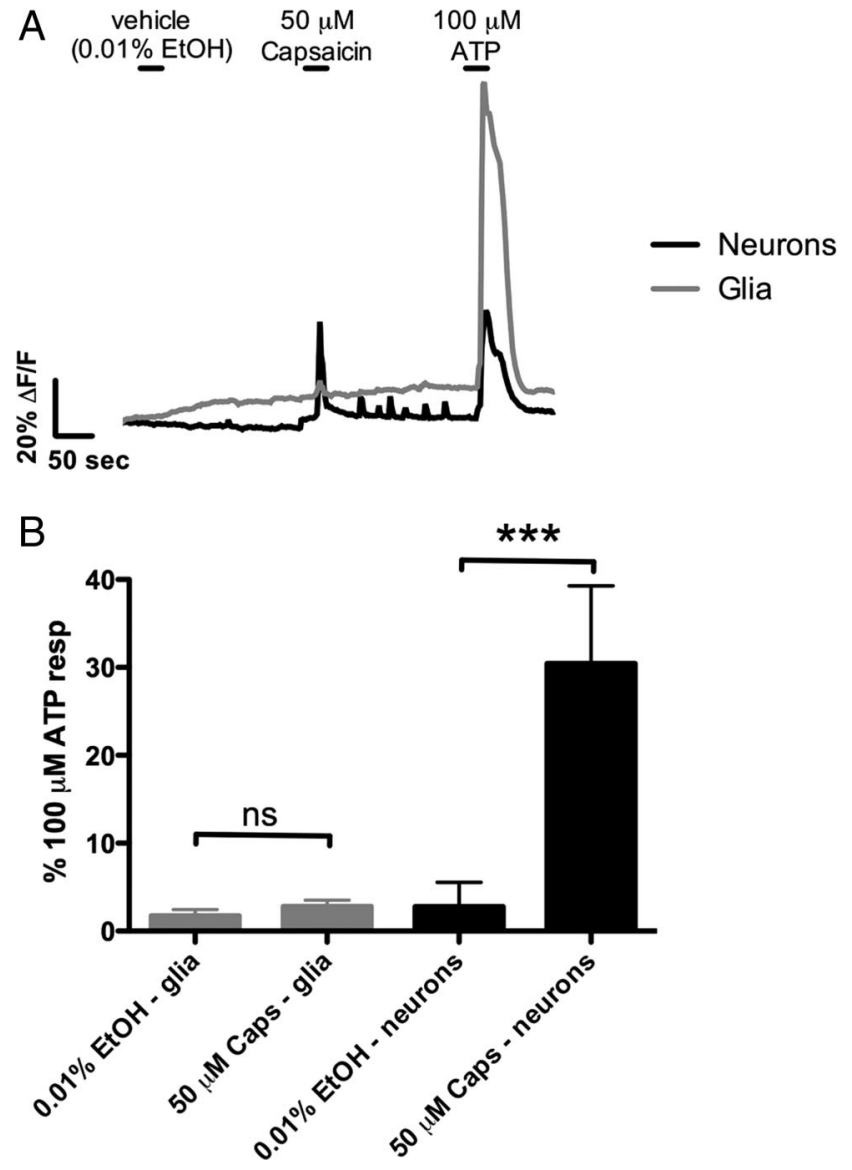

Figure 3. Stimulation of primary afferent nerves with capsaicin stimulates $\mathrm{Ca}^{2+}$ responses in enteric neurons but not enteric glia. $\boldsymbol{A}$, Trace showing the averaged response of enteric glia ( 45 cells; gray) and neurons ( 5 cells; black) within a myenteric ganglion (error bars omitted for clarity). Application of capsaicin $(50 \mu \mathrm{m})$ stimulates $\mathrm{Ca}^{2+}$ responses only in neurons, whereas application of ATP $(100 \mu \mathrm{m})$ elicits responses in both neurons and glia. $\boldsymbol{B}$, Summary data showing that glia (averaged glia response from $n=19$ ganglia) do not respond to capsaicin (ANOVA, $p>0.05$ ). However, capsaicin (Caps) significantly (ANOVA, ${ }^{* *} p<0.0001$ ) stimulates neuronal responses that are $\sim 30 \%$ as large as neuronal ATP responses (averaged neuronal responses from $n=10$ ganglia).

glial activation, we used the neurotoxin 6-OHDA to selectively eliminate noradrenergic neurons. Systemic administration of 6-OHDA destroys the majority of adrenergic terminals in the myenteric plexus of the guinea pig (Qayyum, 1976; Gershon et al., 1980, 1990). In agreement, we find that treatment with 6-OHDA greatly reduces the occurrence of TH-IR nerve fibers within the myenteric plexus (Fig. $4 A$ ). There was however, sparse TH-IR innervation of the myenteric plexus remaining after 6-OHDA treatment, indicating that this treatment was not totally effective in our hands and as previously shown by Gershon et al. (1990).

The myenteric plexus of sympathectomized animals is structurally indistinguishable from control animals, and glial responses to ATP in sympathectomized tissue (mean peak response from $n=23$ ganglia of $0.62 \pm 0.07 \Delta F / F$ ) are not significantly different (two-tailed $t$ test, $p=0.4276$ ) than in control tissue (mean peak response from $n=25$ ganglia of $0.69 \pm 0.07 \Delta F / F$ ). However, sympathectomy significantly reduces the glial FTS $\left[\mathrm{Ca}^{2+}\right]_{\mathrm{i}}$ response amplitude by $\sim 55 \%$ from $32.7 \pm 4.1 \%$ of the ATP amplitude in control tissue ( $n=25$ ganglia, 4 animals) to $18.1 \pm 1.8 \%$ in sympathectomized tissue $(n=24$ ganglia, 3 animals) (two-tailed $t$ test, $p=0.002$ ). Therefore, sympathetic nerve 

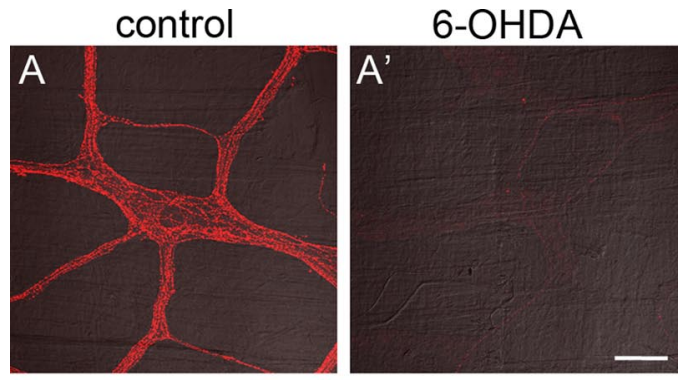

control

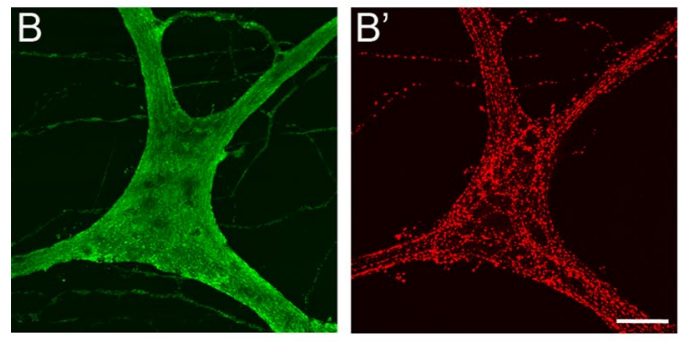

denervated

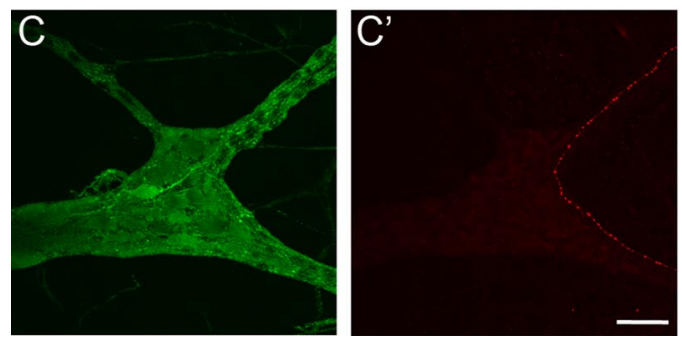

Figure 4. Chemical sympathectomy and extrinsic denervation reduce FTS responses in enteric glia to a similar extent. $\boldsymbol{A}, \boldsymbol{A}^{\prime}$, Chemical sympathectomy. TH-IR sympathetic nerve fibers densely innervate the myenteric plexus of the guinea pig colon under normal conditions $(\boldsymbol{A})$. Treatment with 6-0HDA reduces the number of TH-IR fibers within the myenteric plexus, indicating a loss of sympathetic innervation. Scale bar: $\boldsymbol{A}^{\prime}, 100 \mu \mathrm{m} . \boldsymbol{B}-\boldsymbol{C}^{\prime}$, Surgical extrinsic denervation. In control tissue (taken $\sim 5 \mathrm{~cm}$ oral to the site of denervation), PGP9.5-IR myenteric neurons (green in $\boldsymbol{B}$ ) are surrounded by TH-IR (red in $\boldsymbol{B}^{\prime}$ ) extrinsic fibers. $\boldsymbol{C}, \boldsymbol{C}^{\prime}$, Surgical denervation significantly reduces the amount of TH-IR extrinsic fibers (red in $\boldsymbol{C}^{\prime}$ ), whereas PGP9.5-IR myenteric neurons remain intact (green in $\boldsymbol{C}$ ). Scale bars: $\boldsymbol{B}^{\prime}, \boldsymbol{C}^{\prime}, 50 \mu \mathrm{m}$.

fibers are responsible for a significant component of FTS responses in enteric glia.

\section{Extrinsic denervation}

Treatment with 6-OHDA only removes noradrenergic sympathetic fibers. Because this treatment significantly decreased, but did not totally eliminate, glial FTS responses, we hypothesized that another population of extrinsic nerve fibers (parasympathetic or primary afferent) may contribute to FTS responses in enteric glia. To test this hypothesis, we surgically severed the periarterial nerves innervating a section of colon and assayed the ability of FTS to elicit glial responses after sufficient time for extrinsic nerves to degenerate. Surgical denervation, like 6-OHDA treatment, substantially reduces the occurrence of TH-IR nerve fibers in the myenteric plexus, whereas PGP9.5-IR enteric neurons remain intact (Fig. $4 B, C$ ).

Similarly to 6-OHDA treated tissue, glial FTS $\left[\mathrm{Ca}^{2+}\right]_{\mathrm{i}}$ response amplitude is reduced by $\sim 70 \%$ from $29.5 \pm 2.9 \%$ of the ATP amplitude in control tissue ( $n=15$ ganglia, 4 animals) to $9.1 \pm 1.2 \%$ in denervated tissue $(n=14$ ganglia, 4 animals $)$ (two-tailed $t$ test, $p<0.0001$ ). The glial FTS $\left[\mathrm{Ca}^{2+}\right]_{\mathrm{i}}$ response amplitude in denervated tissue is not significantly different from that in 6-OHDA-treated tissue (ANOVA, $p>0.05$ ), suggesting that other classes of extrinsic nerves do not significantly contribute to glial FTS responses.

Frequency dependence of fiber-tract-stimulated responses in enteric glia

The release of neurotransmitters (ATP vs norepinephrine) from sympathetic varicosities varies with stimulation frequency (Stjarne, 1989; Spencer et al., 1999; Todorov et al., 1999). If enteric glia are activated by sympathetic neurons, activation of enteric glia by FTS should follow the same frequency dependence. Therefore, we tested the effect of varying stimulation frequency on glial activation.

Stimulation at $2 \mathrm{~Hz}$ elicits only rare, small-amplitude responses in enteric glia that are on average $39.5 \pm 5.7 \%$ as large as responses elicited by $20 \mathrm{~Hz}$ stimulation ( $n=7$ ganglia). Increasing stimulation frequency to $10 \mathrm{~Hz}$ stimulates glial responses that are $113.1 \pm 10.6 \%$ as large as responses elicited at $20 \mathrm{~Hz}(n=7)$. Responses elicited by stimulation at $2 \mathrm{~Hz}$ are significantly smaller than those elicited by stimulation at 10 and $20 \mathrm{~Hz}$ (ANOVA, $p<$ 0.0001 ), but responses stimulated at 10 and $20 \mathrm{~Hz}$ are not significantly different (ANOVA, $p>0.05$ ).

\section{Enteric glial responses to sympathetic neurotransmitters}

Our data strongly suggest that sympathetic nerve fibers release substances that stimulate $\left[\mathrm{Ca}^{2+}\right]_{\mathrm{i}}$ responses in enteric glia. Although we demonstrated previously that FTS stimulates a neuronal release of ATP that stimulates enteric glia, other sympathetic neurotransmitters may contribute to glial $\left[\mathrm{Ca}^{2+}\right]_{\mathrm{i}}$ responses. Therefore, we tested whether the sympathetic cotransmitters NE, NPY, and SST could elicit $\left[\mathrm{Ca}^{2+}\right]_{\mathrm{i}}$ responses in enteric glia.

Bath application of NE $(100 \mu \mathrm{M})$ stimulates a pronounced $\left[\mathrm{Ca}^{2+}\right]_{\mathrm{i}}$ response in cells lining blood vessels, followed by vessel constriction (Fig. 5A). NE-induced $\left[\mathrm{Ca}^{2+}\right]_{\mathrm{i}}$ responses also occur in cells (possibly interstitial cells of Cajal) between the myenteric plexus and longitudinal muscle. However, NE fails to elicit $\left[\mathrm{Ca}^{2+}\right]_{\mathrm{i}}$ responses in enteric glia within the myenteric plexus $(n=17$ ganglia from 3 animals) (Fig. 5B). NPY ( $1 \mu \mathrm{M})$ and SST ( 1 $\mu \mathrm{M})$ are also ineffective in stimulating glial $\left[\mathrm{Ca}^{2+}\right]_{\mathrm{i}}$ responses within the myenteric plexus $(n=8$ ganglia from 2 animals for NPY and $n=6$ ganglia from 2 animals for SST) (Fig. 5C,D). NPY-stimulated $\left[\mathrm{Ca}^{2+}\right]_{\mathrm{i}}$ responses do, however, occur in glia within the tertiary plexus (data not shown).

\section{Discussion}

Our observations provide the first evidence that peripheral glia integrate synaptic information. Specifically, our data suggest that enteric glia can discern activity in adjacent neural pathways innervating the myenteric plexus and selectively respond to postganglionic sympathetic neurons. Activation of intrinsic enteric neural circuits does not activate glial $\mathrm{Ca}^{2+}$ responses, and fast enteric neurotransmission is not necessary for initiation of glial FTS responses. However, ablating extrinsic innervation significantly reduces glial $\mathrm{Ca}^{2+}$ responses. The findings that equivalent reduction in glial responses is obtained by selectively ablating sympathetic fibers, glial FTS responses follow the same frequency-dependent profile as neurotransmitter release from sympathetic nerves, and glia are not activated by primary afferent stimulation strongly suggest that sympathetic nerves are primarily responsible for evoked responses in enteric glia. Furthermore, the only sympathetic neurotransmitter that elicits $\mathrm{Ca}^{2+}$ responses in enteric glia is ATP. Our previous findings demonstrate that enteric glial responses to ATP and FTS are mediated by $\mathrm{P}_{2} \mathrm{Y}_{4}$ 


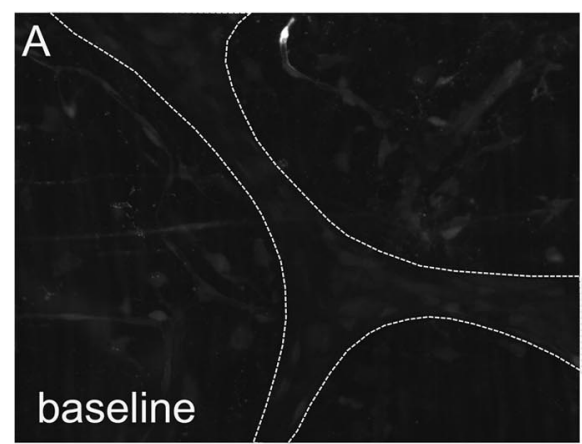

B

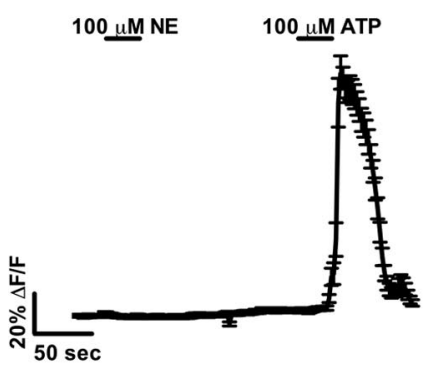

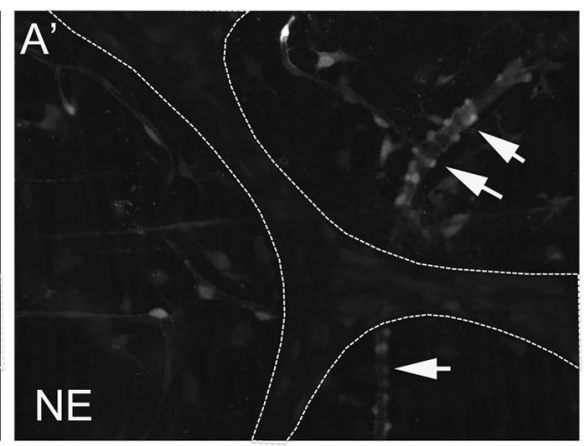

C

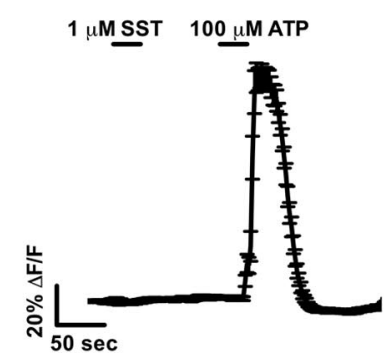

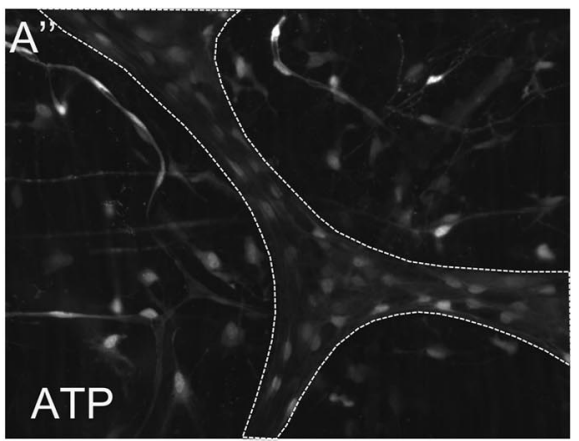

D

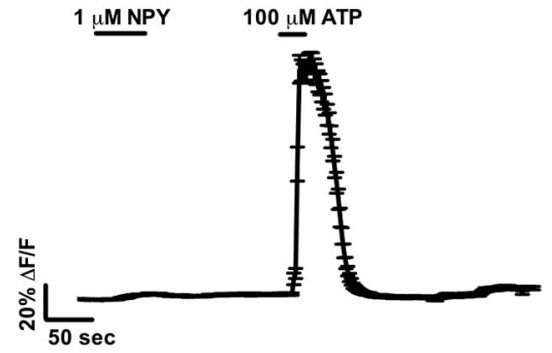

Figure 5. ATP is the only postganglionic sympathetic neurotransmitter that stimulates $\mathrm{Ca}^{2+}$ responses in enteric glia. $A$, Baseline Fluo-4 fluorescence in a myenteric ganglion (outlined by dotted line). $\boldsymbol{A}^{\prime}$, Application of NE $(100 \mu \mathrm{m})$ causes a $\mathrm{Ca}^{2+}$ response in cells lining blood vessels (arrows in $\boldsymbol{A}^{\prime}$ ), resulting in a constriction of the vessel. However, no $\mathrm{Ca}^{2+}$ responses occur in enteric glia within the myenteric ganglia. $A^{\prime \prime}$, Application of ATP $(100 \mu \mathrm{m})$ elicits a robust $\mathrm{Ca}^{2+}$ response in enteric glia. $\boldsymbol{B}$, Trace showing the averaged response (mean \pm SEM) of 35 glial regions within a myenteric ganglion. No responses are detected with application of NE. C, Averaged response (mean \pm SEM) of 34 glial regions within a myenteric ganglion demonstrating that enteric glia do not respond to SST $(1 \mu \mathrm{M})$. D, Enteric glia also do not respond to NPY $(1 \mu \mathrm{m})$. Trace showing averaged response (mean \pm SEM) of 51 glial regions within a myenteric ganglion.

receptors (Gulbransen and Sharkey, 2009). Therefore, we propose a model whereby sympathetic stimulation in the colonic myenteric plexus releases norepinephrine, inhibiting enteric neurons via presynaptic adrenergic receptors (Hirst and McKirdy, 1974) and ATP that acts at stimulatory $\mathrm{P}_{2} \mathrm{Y}_{4}$ receptors on enteric glia (Fig. 6).

Our observations provide new insights into the interactions between autonomic neurons and peripheral glia and provide a novel signaling pathway to control gastrointestinal function. Importantly, we demonstrate that enteric glia are specifically targeted by discrete neuronal populations. Presumably, electrical stimulation activates all neuronal fibers within the interganglionic fiber tracts, and, because ATP is present in all cells (Pankratov et al., 2006), any neuronal component of the ENS has the potential to release ATP. If enteric glia merely serve a supporting role for neurons, it might be expected that general neuronal activity would activate enteric glia. However, modulation of enteric neurotransmission by enteric glia would necessitate that glia detect and differentiate the activity of specific synapses. Functional evidence in the CNS suggests that astrocytes modulate the activity of individual synapses (Gordon et al., 2009). Astrocytes discriminate activity in adjacent neural pathways that use different neurotransmitters (Araque et al. 2002) and the activity of pathways that use common neurotransmitters (Perea and Araque, 2005; Schipke et al. 2008). In the ENS, morphological evidence by Gabella (1981) demonstrates enteric glia are "innervated" by a specific type of vesicle-containing axon, suggesting that enteric glia, like astrocytes, are selectively activated by a specific neural pathway.

An alternate interpretation of our findings is that ATP is not the primary signal from nerves but is in fact a secondary signal used by glia to propagate calcium responses. Much like in CNS astrocytes, propagating calcium waves have been reported in cul- tured enteric glia in response to mechanical stimulation (Zhang et al., 2003). Furthermore, guinea pig enteric glia express mGluR5 receptors, raising the possibility that they may respond to glutamate (Nasser et al., 2007). There are, however, several observations that argue against this scenario in our preparations. First, calcium waves propagating through the enteric glial network have never been observed in situ. In the CNS (either in situ or in vivo), astrocyte calcium responses generated by neuronal activity typically are refined to discrete astrocytes (Schummers et al., 2008; Gordon et al., 2009). In agreement, we find that enteric glial responses to neuronal stimulation do not propagate in a wave-like manner throughout the ganglia or to adjacent ganglia. Second, although guinea pig enteric glia express mGluR5 receptors, stimulation with mGluR agonists (in our hands) does not initiate glial calcium responses (our unpublished observations). In agreement with our findings in situ, Kimball and Mulholland (1996) reported that cultured guinea pig myenteric glia do not respond to glutamate. We also fail to detect enteric glial calcium responses to norepinephrine and muscarinic receptor agonists (our unpublished observation). Although it is possible that another, yet to be identified, neurotransmitter initiates glial calcium responses, we believe that our current data most strongly supports the hypothesis that ATP is responsible for enteric neuronglia signaling.

Nicotinic stimulation only elicited small $\mathrm{Ca}^{2+}$ responses in enteric glia, and nicotinic signaling was not required for evoked glial $\mathrm{Ca}^{2+}$ responses. This was surprising because the majority of fast excitatory neurotransmission in the myenteric plexus is cholinergic and ATP is a cotransmitter at many cholinergic synapses (LePard and Galligan, 1999; Nurgali et al., 2003). White (1982) demonstrated that nicotinic agonists stimulate ATP release from myenteric varicosities. However, when varicosities were isolated from guinea pigs pretreated with 6-OHDA, acetylcholine-evoked 


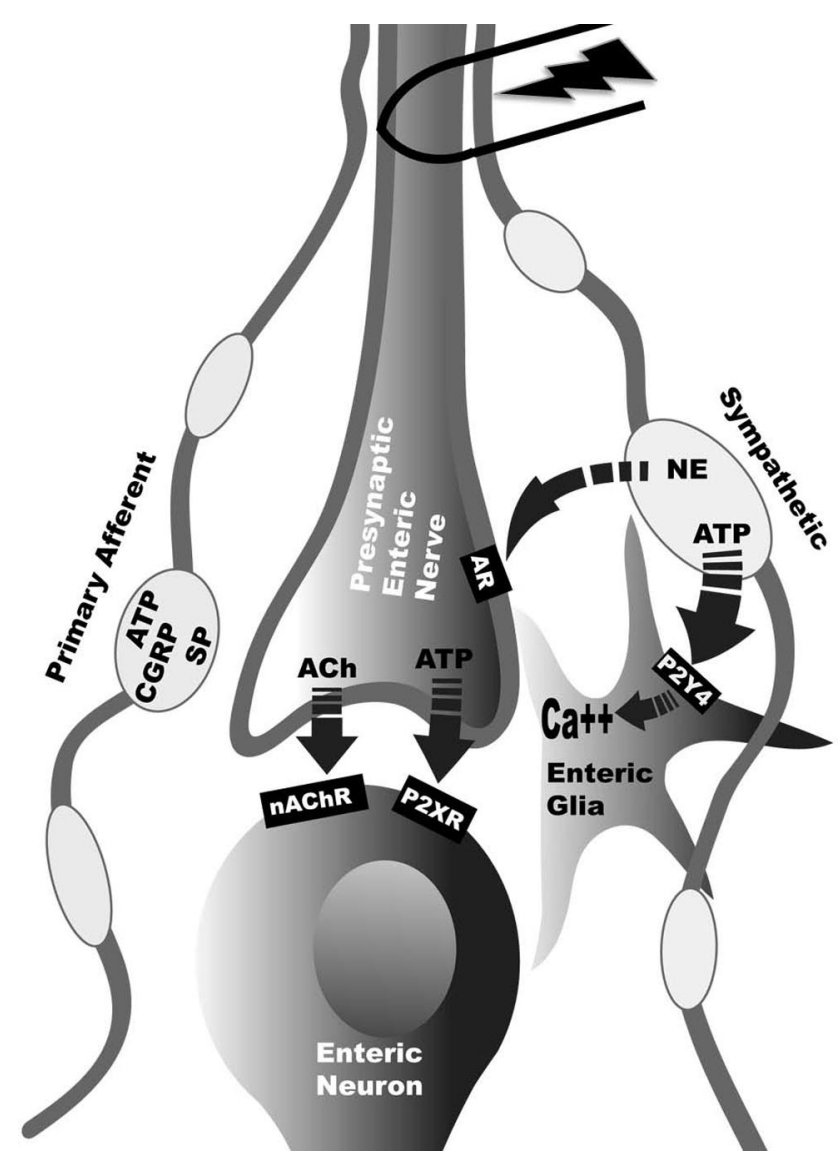

Figure 6. Model of neuron-to-glia transmission in the myenteric plexus of the guinea pig distal colon. Stimulation of primary afferent fibers releases neuropeptides and ATP (CGRP, calcitonin gene-related peptide; SP, substance $P$ ), resulting in stimulation of enteric neurons. Activation of presynaptic enteric neurons release acetylcholine (ACh) and ATP that act on fast nicotinic (nAChR) and purinergic (P2XR) receptors on postsynaptic enteric neurons. Sympathetic activation initially releases ATP that acts at $\mathrm{P}_{2} \mathrm{Y}_{4}$ receptors on enteric glia, eliciting glia $\mathrm{Ca}^{2+}$ responses. The slower sustained sympathetic response releases NE that acts on presynaptic adrenergic receptors (AR) on enteric neurons.

ATP release was drastically reduced (White and Al-Humayyd, 1983). This suggests that much of the ATP release triggered by nicotinic agonists in the myenteric plexus is mediated by sympathetic varicosities and not enteric neurons. Therefore, the small glial responses observed in response to the nicotinic agonist epibatidine may be attributable to sympathetically released ATP.

Our finding that capsaicin did not elevate glial $\mathrm{Ca}^{2+}$ is at odds with the findings of Sharkey et al. (1999) who showed that intraluminal capsaicin induced c-Fos expression in myenteric glia. Although we did not observe glial $\mathrm{Ca}^{2+}$ elevations in response to capsaicin in the present study, this discrepancy is likely attributable to capsaicin stimulating a colo-colonic reflex in vivo through the inferior mesenteric ganglion (IMG) (Maggi et al., 1987; Lomax et al., 2010). Axon collaterals from colonic TRPV1positive primary afferent fibers make excitatory synapses in the guinea pig IMG (Crowcroft et al., 1971). Therefore, in vivo, capsaicin can stimulate the sympathetic supply to the colon, whereas in vitro this circuit is severed.

Chemical sympathectomy with 6-OHDA and surgical denervation of the colon both significantly reduced FTS-induced glial $\mathrm{Ca}^{2+}$ responses. Neither treatment, however, completely eliminated glial responses. The residual responses are likely attributable to incomplete denervation because neither surgical denervation nor
6-OHDA treatment completely eliminated $\mathrm{TH}$ immunoreactivity. An alternate explanation is that another neuronal population (i.e., enteric) does contribute to the enteric glia FTS response and that our current stimulation protocols have failed to adequately stimulate neuron-to-glia signaling in this population. Although our results demonstrate that FTS responses in glia depend on intact sympathetic innervation, we have not demonstrated that selective stimulation of sympathetic neurons stimulates glial $\mathrm{Ca}^{2+}$ responses. The conditions of ATP release in the myenteric plexus are currently unknown, however, and we aim to address this in future studies.

To date, the sympathetic nervous system was thought to primarily innervate glands, muscle, blood vessels, lymphoid tissue, and enteric neurons. In the myenteric plexus of the gastrointestinal tract, sympathetic stimulation releases NE and the cotransmitter ATP (Al-Humayyd and White, 1985). Although NE is thought to inhibit enteric neurons via presynaptic adrenergic receptors (Hirst and McKirdy, 1974; Tack and Wood, 1992; Spencer et al., 1999; Stebbing et al., 2001), the role of sympathetic ATP release is less clearly defined (Venkova and Krier, 1993; Kadowaki et al., 2003). Our results suggest that sympathetic varicosities release ATP to activate enteric glia. Although the physiological consequence of sympathetic neuron-to-enteric glia signaling is unknown, recruitment of glia may be a general feature of noradrenergic signaling throughout the nervous system. Kulik et al. (1999) demonstrated that Bergmann glia in the cerebellum respond to synaptically released noradrenalin. Similar observations were reported after stimulation of the locus ceruleus (Bekar et al., 2008). Therefore, it is tempting to suggest that neuronal responses to stressful situations require glial recruitment. This glial recruitment could function to coordinate the activity of central and enteric neural circuits. In the CNS, astrocytes coordinate network activity by regulating extracellular adenosine. Astrocytederived adenosine exists tonically in the CNS and acts to inhibit neurons presynaptically (Wu and Saggau, 1994). Neuronal activity in a single pathway activates astrocytes, causing adenosine levels to increase and inhibit adjacent nonstimulated pathways (Pascual et al., 2005; Serrano et al., 2006). Similar to CNS adenosine, basal norepinephrine release from sympathetic fibers exerts a tonic inhibitory tone on myenteric neurons (Yuyama et al., 2002). Brief bursts of sympathetic activity initiate fast, transient, ATP release, followed by slower, prolonged, norepinephrine release (Machaly et al., 1988; Todorov et al., 1999). Sympathetic ATP could quickly activate enteric glia, uniformly inhibiting the myenteric plexus before norepinephrine is released. This would be especially important in an organ like the gut in which coordinated output is essential for proper function.

Alternatively, sympathetic neurons may activate enteric glia to sustain enteric neurons during hypoxic periods. Sympathetic stimulation constricts intestinal arterioles, reducing the supply of nutrients and oxygen to enteric neurons (Vanner and Surprenant, 1996). Similar conditions in the CNS stimulate glycolysis and lactate release from astrocytes (Gordon et al., 2008). In the gut, sympathetic stimulation would cause frequent hypoxic periods. Therefore, the enteric nervous system may have evolved this mechanism to sustain neurons during sympathetic stimulation. In support of this hypothesis, myenteric neurons are rapidly lost when sympathetic neurotransmitter release is reduced during gut inflammation (Jacobson et al., 1997; Blandizzi et al., 2003; Linden et al., 2005; Motagally et al., 2009).

In conclusion, enteric glia are a novel target of the sympathetic nervous system. Enteric glia specifically respond to ATP released from sympathetic fibers innervating the myenteric plexus. These 
findings demonstrate that enteric glia, like CNS astrocytes, discriminate the activity of specific neural pathways.

\section{References}

Al-Humayyd M, White TD (1985) Adrenergic and possible nonadrenergic sources of adenosine 5 -triphosphate release from nerve varicosities isolated from ileal myenteric plexus. J Pharmacol Exp Ther 233:796-800.

Araque A, Martín ED, Perea G, Arellano JI, Buño W (2002) Synaptically released acetylcholine evokes $\mathrm{Ca}^{2+}$ elevations in astrocytes in hippocampal slices. J Neurosci 22:2443-2450.

Bekar LK, He W, Nedergaard M (2008) Locus coeruleus alpha-adrenergicmediated activation of cortical astrocytes in vivo. Cereb Cortex 18:2789-2795.

Blandizzi C, Fornai M, Colucci R, Baschiera F, Barbara G, De Giorgio R, De Ponti F, Breschi MC, Del Tacca M (2003) Altered prejunctional modulation of intestinal cholinergic and noradrenergic pathways by alpha2adrenoceptors in the presence of experimental colitis. Br J Pharmacol 139:309-320.

Brierley SM, Carter R, Jones W 3rd, Xu L, Robinson DR, Hicks GA, Gebhart GF, Blackshaw LA (2005) Differential chemosensory function and receptor expression of splanchnic and pelvic colonic afferents in mice. J Physiol 567:267-281.

Burnstock G (2009) Purinergic cotransmission. Exp Physiol 94:20-24.

Crowcroft PJ, Holman ME, Szurszewski JH (1971) Excitatory input from the distal colon to the inferior mesenteric ganglion in the guinea-pig. J Physiol 219:443-461.

Furness JB (2006) The enteric nervous system. Malden, MA: Blackwell.

Gabella G (1981) Ultrastructure of the nerve plexuses of the mammalian intestine: the enteric glial cells. Neuroscience 6:425-436.

Galligan JJ, Bertrand PP (1994) ATP mediates fast synaptic potentials in enteric neurons. J Neurosci 14:7563-7571.

Gershon MD, Sherman DL, Dreyfus CF (1980) Effects of indolic neurotoxins on enteric serotonergic neurons. J Comp Neurol 190:581-596.

Gershon MD, Sherman DL, Pintar JE (1990) Type-specific localization of monoamine oxidase in the enteric nervous system: relationship to 5-hydroxytryptamine, neuropeptides, and sympathetic nerves. J Comp Neurol 301:191-213.

Gomes P, Chevalier J, Boesmans W, Roosen L, van den Abbeel V, Neunlist M, Tack J, Vanden Berghe P (2009) ATP-dependent paracrine communication between enteric neurons and glia in a primary cell culture derived from embryonic mice. Neurogastroenterol Motil 21:870-e62.

Gordon GR, Choi HB, Rungta RL, Ellis-Davies GC, MacVicar BA (2008) Brain metabolism dictates the polarity of astrocyte control over arterioles. Nature 456:745-749.

Gordon GR, Iremonger KJ, Kantevari S, Ellis-Davies GC, MacVicar BA, Bains JS (2009) Astrocyte-mediated distributed plasticity at hypothalamic glutamate synapses. Neuron 64:391-403.

Gulbransen BD, Sharkey KA (2009) Purinergic neuron-to-glia signaling in the enteric nervous system. Gastroenterology 136:1349-1358.

Hanani M, Reichenbach A (1994) Morphology of horseradish peroxidase (HRP)-injected glial cells in the myenteric plexus of the guinea-pig. Cell Tissue Res 278:153-160.

Hirst GD, McKirdy HC (1974) Presynaptic inhibition at mammalian peripheral synapse? Nature 250:430-431.

Jacobson K, McHugh K, Collins SM (1997) The mechanism of altered neural function in a rat model of acute colitis. Gastroenterology 112:156-162.

Jessen KR, Mirsky R (1983) Astrocyte-like glia in the peripheral nervous system: an immunohistochemical study of enteric glia. J Neurosci 3:2206-2218

Kadowaki M, Yoneda S, Takaki M (2003) Involvement of a purinergic pathway in the sympathetic regulation of motility in rat ileum. Auton Neurosci 104:10-16.

Kettenmann H, Ransom B (2004) Neuroglia. New York: Oxford UP.

Kimball BC, Mulholland MW (1996) Enteric glia exhibit P2U receptors that increase cytosolic calcium by a phospholipase C-dependent mechanism. J Neurochem 66:604-612.

Kulik A, Haentzsch A, Lückermann M, Reichelt W, Ballanyi K (1999) Neuron-glia signaling via $\alpha_{1}$ adrenoceptor-mediated $\mathrm{Ca}^{2+}$ release in Bergmann glia cells in situ. J Neurosci 19:8401-8408.

LePard KJ, Galligan JJ (1999) Analysis of fast synaptic pathways in the myenteric plexus of guinea pig ileum. Am J Physiol 276:G529-G538.

Linden DR, Couvrette JM, Ciolino A, McQuoid C, Blaszyk H, Sharkey KA,
Mawe GM (2005) Indiscriminate loss of myenteric neurones in the TNBS-inflamed guinea-pig distal colon. Neurogastroenterol Motil 17:751-760.

Lomax AE, Zhang JY, Furness JB (2000) Origins of cholinergic inputs to the cell bodies of intestinofugal neurons in the guinea pig distal colon. J Comp Neurol 416:451-460.

Lomax AE, Sharkey KA, Furness JB (2010) The participation of the sympathetic innervation of the gastrointestinal tract in disease states. Neurogastroenterol Motil 22:7-18.

Machaly M, Dalziel HH, Sneddon P (1988) Evidence for ATP as a cotransmitter in dog mesenteric artery. Eur J Pharmacol 147:83-91.

Maggi CA, Meli A, Santicioli P (1987) Four motor effects of capsaicin on guinea-pig distal colon. Br J Pharmacol 90:651-660.

Malin SA, Christianson JA, Bielefeldt K, Davis BM (2009) TPRV1 expression defines functionally distinct pelvic colon afferents. J Neurosci 29:743-752.

Messenger JP, Furness JB (1992) Distribution of enteric nerve cells that project to the coeliac ganglion of the guinea-pig. Cell Tissue Res 269:119-132.

Motagally MA, Neshat S, Lomax AE (2009) Inhibition of sympathetic $\mathrm{N}$-type voltage-gated $\mathrm{Ca}^{2+}$ current underlies the reduction in norepinephrine release during colitis. Am J Physiol Gastrointest Liver Physiol 296:G1077-G1084.

Nasser Y, Keenan CM, Ma AC, McCafferty DM, Sharkey KA (2007) Expression of a functional metabotropic glutamate receptor 5 on enteric glia is altered in states of inflammation. Glia 55:859-872.

Nurgali K, Furness JB, Stebbing MJ (2003) Analysis of purinergic and cholinergic fast synaptic transmission to identified myenteric neurons. Neuroscience 116:335-347.

Olsson C, Chen BN, Jones S, Chataway TK, Costa M, Brookes SJ (2006) Comparison of extrinsic efferent innervation of guinea pig distal colon and rectum. J Comp Neurol 496:787-801.

Pankratov Y, Lalo U, Verkhratsky A, North RA (2006) Vesicular release of ATP at central synapses. Pflugers Arch 452:589-597.

Pascual O, Casper KB, Kubera C, Zhang J, Revilla-Sanchez R, Sul JY, Takano H, Moss SJ, McCarthy K, Haydon PG (2005) Astrocytic purinergic signaling coordinates synaptic networks. Science 310:113-116.

Perea G, Araque A (2005) Properties of synaptically evoked astrocyte calcium signal reveal synaptic information processing by astrocytes. J Neurosci 25:2192-2203.

Qayyum MA (1976) Fluorescence histochemical study of Aurbach's plexus of the ileum of 6-hydroxydopamine-treated guinea pig. Acta Anat (Basel) 96:497-512.

Robinson DR, McNaughton PA, Evans ML, Hicks GA (2004) Characterization of the primary spinal afferent innervation of the mouse colon using retrograde labelling. Neurogastroenterol Motil 16:113-124.

Schipke CG, Haas B, Kettenmann H (2008) Astrocytes discriminate and selectively respond to the activity of a subpopulation of neurons within the barrel cortex. Cereb Cortex 18:2450-2459.

Schummers J, Yu H, Sur M (2008) Tuned responses of astrocytes and their influence on hemodynamic signals in the visual cortex. Science 320:1638-1643.

Serrano A, Haddjeri N, Lacaille JC, Robitaille R (2006) GABAergic network activation of glial cells underlies hippocampal heterosynaptic depression. J Neurosci 26:5370-5382.

Sharkey KA, Parr EJ, Keenan CM (1999) Immediate-early gene expression in the inferior mesenteric ganglion and colonic myenteric plexus of the guinea pig. J Neurosci 19:2755-2764.

Spencer N, McCarron SL, Smith TK (1999) Sympathetic inhibition of ascending and descending interneurones during the peristaltic reflex in the isolated guinea-pig distal colon. J Physiol 519:539-550.

Stebbing M, Johnson P, Vremec M, Bornstein J (2001) Role of alpha(2)adrenoceptors in the sympathetic inhibition of motility reflexes of guineapig ileum. J Physiol 534:465-478.

Stjärne L (1989) Basic mechanisms and local modulation of nerve impulseinduced secretion of neurotransmitters from individual sympathetic nerve varicosities. Rev Physiol Biochem Pharmacol 112:1-137.

Tack JF, Wood JD (1992) Actions of noradrenaline on myenteric neurons in the guinea pig gastric antrum. J Auton Nerv Syst 41:67-77.

Takahashi A, Camacho P, Lechleiter JD, Herman B (1999) Measurement of intracellular calcium. Physiol Rev 79:1089-1125. 
Todorov LD, Mihaylova-Todorova ST, Bjur RA, Westfall DP (1999) Differential cotransmission in sympathetic nerves: role of frequency of stimulation and prejunctional autoreceptors. J Pharmacol Exp Ther 290:241-246.

Vanner S, Surprenant A (1996) Neural reflexes controlling intestinal microcirculation. Am J Physiol 271:G223-G230.

Venkova K, Krier J (1993) Stimulation of lumbar sympathetic nerves evokes contractions of cat colon circular muscle mediated by ATP and noradrenaline. Br J Pharmacol 110:1260-1270.

White TD (1982) Release of ATP from isolated myenteric varicosities by nicotinic agonists. Eur J Pharmacol 79:333-334.

White TD, Al-Humayyd M (1983) Acetylcholine releases ATP from varicos- ities isolated from guinea pig myenteric plexus. J Neurochem 40:10691075.

Wu LG, Saggau P (1994) Adenosine inhibits evoked synaptic transmission primarily by reducing presynaptic calcium influx in area CA1 of hippocampus. Neuron 12:1139-1148.

Yuyama N, Mizuno J, Tsuzuki H, Wada-Takahashi S, Takahashi O, Tamura K (2002) Effects of extrinsic autonomic inputs on expression of c-Fos immunoreactivity in myenteric neurons of the guinea pig distal colon. Brain Res 948:8-16.

Zhang W, Segura BJ, Lin TR, Hu Y, Mulholland MW (2003) Intercellular calcium waves in cultured enteric glia from neonatal guinea pig. Glia 42:252-262. 